\title{
SynapticDB, Effective Web-based Management and Sharing of Data from Serial Section Electron Microscopy
}

\author{
Bitao Shi • Jennifer Bourne • Kristen M. Harris
}

Published online: 22 December 2010

(C) The Author(s) 2010. This article is published with open access at Springerlink.com

\begin{abstract}
Serial section electron microscopy (ssEM) is rapidly expanding as a primary tool to investigate synaptic circuitry and plasticity. The ultrastructural images collected through ssEM are content rich and their comprehensive analysis is beyond the capacity of an individual laboratory. Hence, sharing ultrastructural data is becoming crucial to visualize, analyze, and discover the structural basis of synaptic circuitry and function in the brain. We devised a web-based management system called SynapticDB (http://synapses.clm.utexas.edu/synap $\mathrm{ticdb} /$ ) that catalogues, extracts, analyzes, and shares experimental data from ssEM. The management strategy involves a library with check-in, checkout and experimental tracking mechanisms. We developed a series of spreadsheet templates (MS Excel, Open Office spreadsheet, etc) that guide users in methods of data collection, structural identification, and quantitative analysis through ssEM. SynapticDB provides flexible access to complete templates, or to individual columns with instructional headers that can be selected to create user-defined templates. New templates can also be generated and uploaded. Research progress is tracked via experimental note management and dynamic PDF forms that allow new investigators to follow standard protocols and experienced researchers to expand the range of data collected and shared. The combined use of templates and tracking notes
\end{abstract}

\footnotetext{
B. Shi $\cdot$ J. Bourne $\cdot$ K. M. Harris $(\bowtie)$

Center for Learning and Memory,

The University of Texas at Austin,

University Station C7000,

Austin, TX 78712-0805, USA

e-mail: kharris@mail.clm.utexas.edu
}

ensures that the supporting experimental information is populated into the database and associated with the appropriate ssEM images and analyses. We anticipate that SynapticDB will serve future meta-analyses towards new discoveries about the composition and circuitry of neurons and glia, and new understanding about structural plasticity during development, behavior, learning, memory, and neuropathology.

Keywords Online database systems · Online data file checkout and check-in · Data and image sharing - Synapse structure and function - Data management - Dynamic PDF forms

\section{Introduction}

Vast improvements in computer science and informatics have resulted in the development of diverse neuroscience database systems (http://neuinfo.org/) (Amari et al. 2002; Bjaalie and Leergaard 2005; Davison et al. 2004; Gardner et al. 2008; Liu et al. 2004; Martone et al. 2003; Nielsen et al. 2004; Pittendrigh and Jacobs 2003; Toga 2002). One purpose for building web-accessible neuroscience database systems is to share data remotely (Martone et al. 2004; Van Horn et al. 2004). This goal is especially true for ssEM images, which require substantial expertise to obtain, yet are rich in detail that cannot be fully analyzed by a single research laboratory. Previous structural databases have focused on molecular, cellular, and regional organization of the brain (Laird et al. 2005; Li et al. 2002; Rosen et al. 2003). Some databases also contain models of structure-function relationships based on whole cell and dendritic levels (Liu et al. 2004; Davison et 
al. 2004; http://neuromorpho.org), while others have incorporated physiological and behavioral data (Crabbe and Morris 2004; Gardner et al. 2005; Tecott and Nestler 2004). As yet, no database exists for the ultrastructure of the nervous system obtained through ssEM. The goal for such a database is to have a management interface that allows investigators to share the same images and avoid unintended redundancy in reconstruction effort. Also needed is a routine means to share experimental conditions in a flexible and portable way. Finally, the ssEM images in the database need to be structured and managed so that future meta-analyses are interpretable.

Our first goal has been to develop a management system to upload large volumes of ssEM images and organize them logically for reuse. The second goal is to facilitate ongoing analysis and discovery without duplication of effort. Towards this goal we have developed a library check-in, checkout and tracking mechanism to expand and share ssEM images and associated reconstruction elements including traces, 3D visualizations, and calibrated quantification of the reconstructed objects. Templates have been established for routine analysis across ssEM images of dendrites, axons, spines, synapses, glial processes, and subcellular core structures, such as polyribosomes and smooth endoplasmic reticulum, as well as other components of the neuropil including extracellular space. These templates have been populated into the database. In addition, there is a tool to create new templates that are readily accessible to the database, without having to rewrite the underlying code. We have tested the system in our laboratory by uploading 26 sets of ssEM images, traces, templates, and metadata that we continue to use for ongoing research. Each set has at least 3 versions of reconstructions that have been checked in and out and cured by more than one user. In addition, we have made public 3 sets of images with associated metadata; and we plan to increase this number as we assemble the metadata from our publications and complete ongoing studies. SynapticDB provides a new environment in which to explore and analyze data rich images of brain tissue at the ultrastructural, i.e. synaptic, level of resolution.

\section{Database Methods and Sample Content}

A powerful database system will integrate experimental elements, store large volumes of data, and provide intelligent interfaces to manage and monitor the status of projects with easy access to existing data for reuse in new studies. We designed and built a web-accessible relational database system called "SynapticDB" (http://synapses.clm.utexas.edu/synapticdb/). It is used to store and share our large volumes of ssEM images, their associated experimental conditions and analyses. Its data repository center uses a relational database and file repository to accommodate the ssEM images and associated data. To enhance data management and transactions from investigations using ssEM images, there are various tools to upload and update, to download and query, and to analyze and search all images, experimental notes and data. In addition there is a User Guide and a list of answers to frequently asked questions.

Data Sources and Management in the Designed Entity Relational Database

The first step was to organize the various data sources and provide flexible ways to enter different types of data and files into the database (Fig. 1). Data sets are comprised of uniquely named experiments that contain all of the information regarding each experiment, including detailed notes about the electrophysiology conditions, tissue processing, serial sectioning, acquisition of ssEM images, and quantitative analyses (Fig. 1a). In our lab, RECONSTRUCT ${ }^{\mathrm{TM}}$ (Fiala and Harris 2001; Fiala 2005; http://synapses.clm.utexas.edu/ tools/reconstruct/reconstruct.stm) is used to extract and save quantitative data from the ssEM images into organized spreadsheets and to produce $3 \mathrm{D}$ reconstructions of the objects. SynapticDB does not require that the input be specifically in the RECONSTRUCT ${ }^{\mathrm{TM}}$ format; hence, files from other reconstruction software systems, such as 3D Doctor (http://www.ablesw.com) or Amira (http:// www.amira.com/), could be used to populate the database equally well at this step. There are several classes of data loaded including: dynamic PDF forms; spreadsheets; scanned notes and drawings; electronic word documents; original physiological recordings; original and processed ssEM images; 3D objects in various formats, and analyses at multiple levels using various statistical packages (Fig. 1b).

The data repositories are composed of two parts to accommodate both direct data entry and uploading of files (Fig. 1c). Retrievable data and information from data files can be populated into the SynapticDB tables. The files with drawings, images or pictures are saved on the server hard drive with the relevant file links (pointers) stored in the database. This structure provides flexibility to the experimenter in the types of data that can be linked to the database.

SynapticDB was designed to maintain proper relationships between many different types of data. Independent interfaces to SynapticDB are organized around a set of Data Transaction Managers that control and manage access to Data and File Repositories (Fig. 2a). The Project Status Interfaces provide an integrated view of 


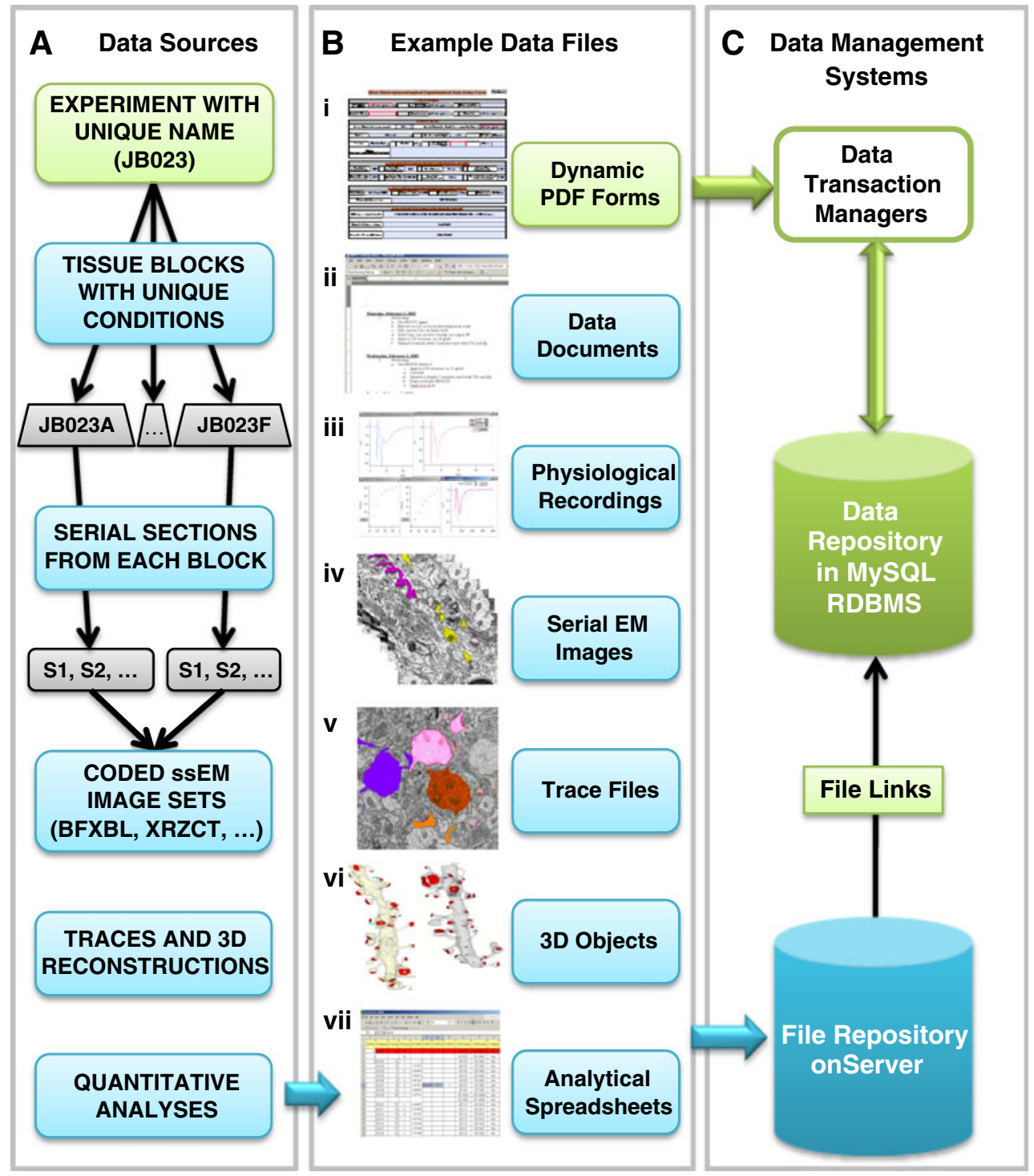

Fig. 1 Data sources, data composition, and the structure of SynapticDB. a Schematic overview of Data Sources from a typical experiment organized into the hierarchical relationships identified by unique experiment, tissue block, and coded image names. These relationships are the basis of the database system. b Example types of data files that are uploaded into the database: (i) Dynamic PDF forms collect experimental notes and data that can be populated into and retrieved from the database; (ii) Data documents are files that include Word documents, PDF files, scanned files, with drawings, notes, etc. describing the experimental procedures or other aspects of what was done to collect the data; (iii) Output from specific electrophysiological recording software (e.g. IGOR) in the original data format; (iv)

the entire experiment and instantly populate the database with experimental notes and data. The Library Check-in and Check-out Interfaces control investigator access to specific series. The Instructional Interfaces include templates with guides for standardized identification and quantification of structures. The Data Sharing Interfaces provide access to data and images via unique investigator names, experiment names, and tissue block or code names,
Original source and compressed ssEM image files, (v) Trace files from RECONSTRUCT ${ }^{\mathrm{TM}}$, or other programs, used to define objects across serial sections; (vi) Reconstructed 3D objects in snapshots or movie formats for visualization of dendrites with spines and synapses, axons, glia etc. (vii) Analytical spreadsheets (e.g. from MS Excel, Statistica) containing electrophysiological waveforms; ultrastructural object identifications; quantitative measurements, statistical analyses, etc. c Experimental parameters and data from the dynamic PDF data collection forms are populated into the database. All other files are stored on the server hard drive and their respective File Links are stored in the database

so that investigators can remain blind as to experimental conditions while performing analyses.

The tables in SynapticDB were created and implemented in the MySQL (http://www.mysql.com ) relational database management system (RDBMS) based on a designed entity relational diagram (ERD) and hosted on a Windows server (Fig. 2b). The designed SynapticDB ERD has several independent parts that provide users with flexible options for 

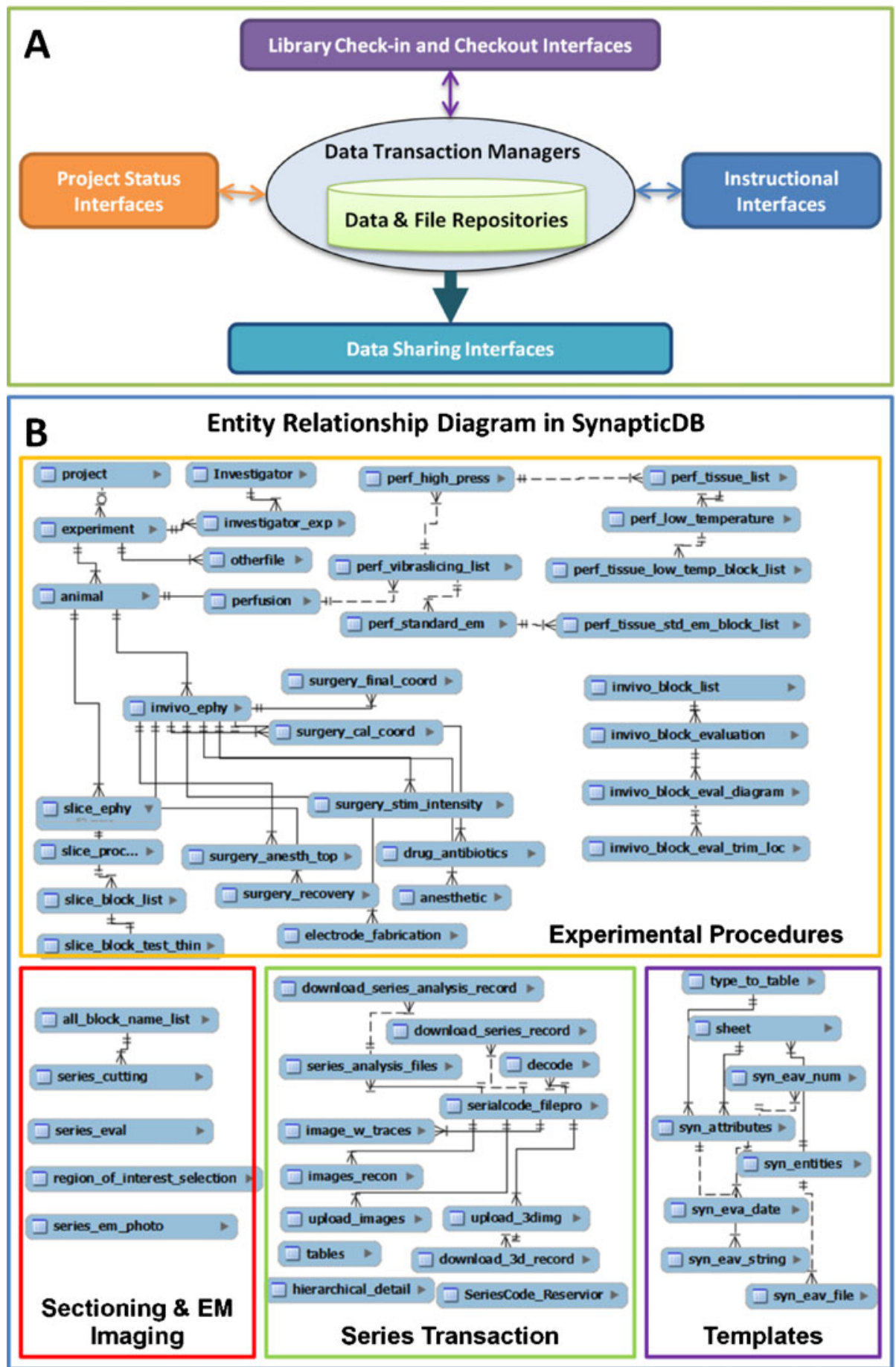

Fig. 2 Overview of SynapticDB. a Diagrammatic representation of the four active sets of web interfaces. Library Check-in and checkout interfaces provide transactions to manage ssEM images and RECONSTRUCT ${ }^{\mathrm{TM}}$ trace files or trace files from other programs used to identify particular objects in the images. Project status interfaces monitor the progress and provide information about which experimental notes and conditions have been integrated on the database. Instructional interfaces build, store, modify and share templates for data analyses. Data sharing interfaces allow querying of the entered data to locate specific experiments or to identify specific types of data available across experiments. All interfaces are associated with their corresponding data transaction managers to organize and manage the data in the data and file repositories. b The entity relationship diagram (ERD) in SynapticDB models the structure of the experimental data sources shown in Fig. 1a. The ERD is implemented in the MySQL Relational Database Management System (RDBMS). The orange box labeled "Experimental Procedures" models the procedures from initial experiments through tissue block processing and evaluation. The red box labeled "Sectioning and EM Imaging" represents input regarding notes for serial ultrathin sectioning and staining, and for evaluation of test thins for tissue quality and final imaging of the serial section. The green box labeled "Series Transaction" is the part of the ERD that is designed to record all series transactions stored in the database. The purple box labeled "Templates" is for managing the portion of the database that is used to access and create analysis templates as discussed further below 
uploading data and files. For example, users can upload sets of ssEM images without first having to upload their experimental conditions or vice versa - at they may enter data from an electrophysiology experiment prior to entering the tissue processing data. Ultimately, the integrating these independent parts in the database are controlled by Data Transaction Managers.

Managing the Data Acquisition and Population in SynapticDB

Data acquisition and population are essential in any database system. Most of the data populated into SynapticDB are experimental conditions and analytical information associated with ssEM images. Those data are not only integrated with the uploaded ssEM images, but are also used to reflect the status of each experiment. SynapticDB manages those experimental notes and data - using dynamic PDF data collection forms. After a unique experimental name is uploaded, the status of uploading the remaining components of the project can be checked and monitored under that experimental name.. Progress in data entry can be monitored when the experimental name is invoked (Fig. 3), and a check mark indicates whether particular data have been uploaded (Fig. 4). If data have been uploaded, then there is a hyperlink allow access to the data, for example, "view data or download or update serial images" (Fig. 4). Otherwise, the raised PDF logo provides a hyperlink to a downloadable dynamic data collection form that the users can fill in for that step.

The dynamic PDF forms for data collection were designed with Adobe LiveCycle Designer ES 8.2 software and their pre-population capacities were implemented based on iText API (http://itextpdf.com/). Creating an efficient mechanism to manage the diversity of data collected during our experiments was initially perplexing. Forms were needed that could populate the database while retaining portability and flexibility to expand and accommodate differing amounts of data. PDF forms were created by using Adobe LiveCycle Designer with these specific features of portability and flexibility (Nesvold 2007) satisfied our requirements. The dropdown boxes on these forms are pre-populated with existing key names, such as experiment and block names, with the flexibility to add new in the fillable boxes which are later added to the database once the forms are uploaded, and populated into subsequent dropdown menus (Fig. 5a, b). The dynamic PDF data forms can be filled in at any time and saved on any machine and then later submitted online (blue arrow, Fig. 5b). Once submitted, the entered data are sent to a Java servlet via the embedded url in the PDF form submit button. Then an xml parsing application parses received data and populates them into the database tables. To accommodate different amounts of data, forms were designed to allow flexibility; a single click adds tables (Fig. 5a, b) or rows to an existing table (Fig. 5c, d). In this way, unforeseen data collection requirements are readily met without requiring adjustment to the underlying structure of SynapticDB.

\section{Library Checkout and Check-in Mechanisms}

Managing large volumes of ssEM images and their analytic information is one of the most important features in SynapticDB. It involves the uploading, downloading and updating of transactions as well as storage and sharing of data. The library checkout and check-in mechanisms were created to use SynapticDB to track transactions and to avoid unwanted duplication of effort on particular datasets. Figure 6 shows an example transaction to checkout a set of ssEM images from SynapticDB. First, the code name is selected (Fig. 6a). Then the most recent version of the file is identified by a name, which consists of that code, the name of the investigator who last contributed to the dataset, and the date and time that the dataset was uploaded (Fig. 6b). The user can check out the dataset by clicking submit to download (Fig. 6c). There is also an option to view a high resolution image of the central EM section in the image series of the file. If the dataset is being used within our laboratory then a locking mechanism is set. If the data are public, then a list is created of who has checked out the data with their entered statement of intended use. There is no locking mechanism on data sets that are in the public domain.

For use within our laboratory, SynapticDB sets the 'in use' icon and sets the locking option on the dataset at the time of checkout to prevent multiple users from simultaneously accessing the same data and accidentally duplicating reconstruction and analysis efforts (Fig. 7a). The "UPDATE (CHECK-IN)" hyperlink allows the current user to check-in and unlock the dataset making it available to other users (Fig. 7b). The "get the user information" hyperlink provides information about the individual who currently has the data checked out (Fig. 7c). If other investigators want to use data that are checked out, they may contact the current user or the system administrator for access. There is also an option to upload new serial EM images in this same area with protections that ensure the data are linked to appropriate experimental data and properly coded (Fig. 7b) by sending the user back to the upload new data stream illustrated in Fig. 3 above. At present, SynapticDB does not have the capacity to manage images uploaded from the Public.

The check-in and update interfaces were implemented from a Java Applet (JUpload Component: http:/www. jupload.biz) that provides flexibility to upload multiple files at once (Fig. 8). This interface adds files from the local machine to the Applet (Fig. 8a), and then uploads new files, 


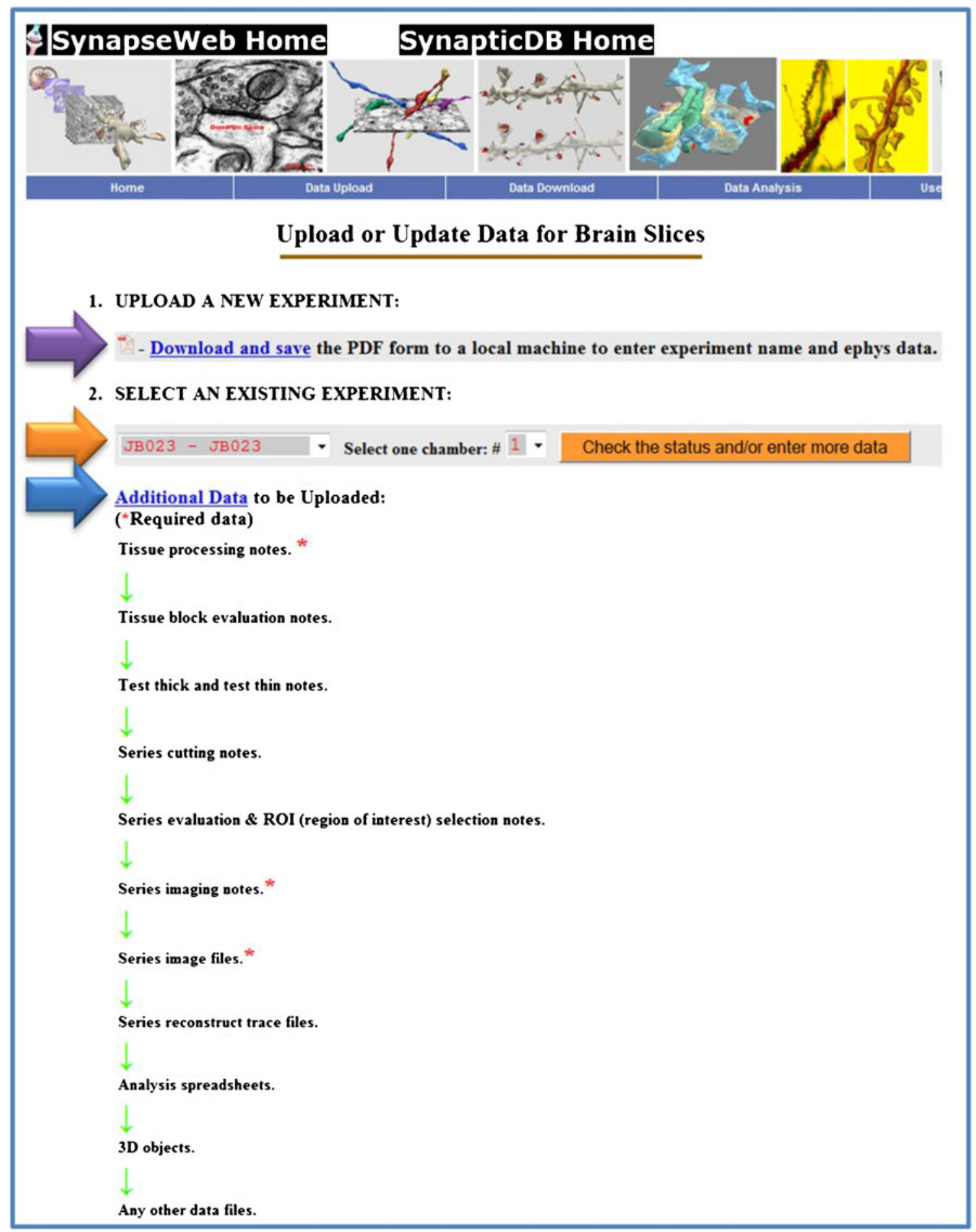

Fig. 3 Interactive webpage manages the upload and update of experimental data. First, an experiment is named and initial data are uploaded into the database (purple arrow). Then the status of data entry for an existing experiment can be checked (orange arrow), or data can be added or updated. The data acquisition

or updates existing files from the Applet to the server (Fig. 8b). Multiple files can be compressed into one zip file on-the-fly and stored on the server. Relevant links to these files are maintained in the database tables by file links (see checklist is listed in the order that the data are typically acquired (blue arrow). Note that only three data sets are required in SynapticDB, namely the Tissue Processing Notes, Series Imaging Notes, and the serial EM images files themselves

Fig. 2c above). Trace files from a program like RECONSTRUCT ${ }^{\mathrm{TM}}$ are routinely managed by the checkout and check-in library mechanism to add, edit, and update datasets (Fig. 9). For example, in our experiments, if ten 
Fig. 4 Overview of data entry for an example experiment. The green checked boxes represent data that had been uploaded to SynapticDB. Where data exist the user is given the options: to "view data", to "upload/update data files", to obtain a list of "expected files" for each step, or to hyperlink to the "protocol" that explains in detail the methods used to collect that particular set of data. The red unchecked boxes indicate that the data for those experimental steps have not been uploaded. The PDF logo provides a hyperlink to download the appropriate PDF form for data entry

\section{Experiment: JB023 - Chamber \#1: Checklist}

Note: ㅍ: Existing Data; $\square$ : No data entered; ${ }^{*}$ Required data;

Download, save and fill in the PDF forms in a local machine to upload data.

1. Exp, Animal \& Ephys Info: view data ; upload/update data files; list expected files; protocol

2. Tissue Processing Notes: view data ; upload/update the data file; list expected files; protocol $\downarrow$

3. Tissue Block Evaluation Info: protocol; list expected uploading files

○ Block: JB023A - - Upload the tissue block evalutation notes.

○ Block: JB023B - - Upload the tissue block evalutation notes.

- $\square$ Block: JB023C - - Upload the tissue block evalutation notes.

$\downarrow$

4. Block Test Thick \& Thin Info: protocol; list expected uploading files

$\circ \square$ Block: JB023A - - Upload the test thick and test thin notes.

○ Block: JB023B - - - Upload the test thick and test thin notes.

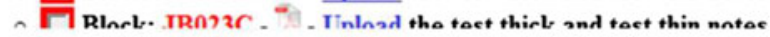

Slock Tissue Series Cutting Info: protocol; list expected uploading files

$\circ \square^{-}$- Upload more series cutting notes.

$\downarrow$

6. Series Evaluation \& Region Selection Info: protocol; list expected uploading files ○ - Upload series evaluation notes.

7. Series EM Imaging Notes: (code a series set) protocol

$\circ \square^{-}$- Upload series imaging notes - *

8. Series Image Uploading Info:

- V Code: FPNCT - download serial images; update the checked out serial images.

- 7 Code: QFBQF - download serial images; update the checked out serial images.

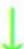

9. Reconstruction Trace Uploading Info:

- $\mid$ Code: BFXBL - download series reconstruct trace files; update the checked out traces.

- $\checkmark$ Code: FPNCT - download series reconstruct trace files; update the checked out traces. $\downarrow$

10. Analysis Spreadsheet Uploading Info:

$\circ \square$ - Upload/update analysis spreadsheets.

$\downarrow$

11. Synaptic 3D Object File Uploading Info:

- Code: FPNCT - Upload 3D object files.

- $\square$ Code: QFBQF - Upload 3D object files.

12. $\square$ Any Other Data Files: $\underline{\text { Upload }}$ different dendrites need to be reconstructed from two different sets of serial images by two different users, then each user would trace five dendrites in each of the series to balance subjective differences in tracing style across the two series. The library mechanism ensures that all trace files are backed up, and that the most up-to-date trace files can be readily identified to avoid duplication of effort. If a user wishes instead to return to an older version of the traces, or to compare trace quality between datasets, it is also possible to download an older version of the dataset by selecting it from the list.

Analysis Templates

Standardizing ssEM image analysis promotes systematic data acquisition and sharing, while reducing interpretive errors. SynapticDB provides an instructive template management platform to list, add, build and modify data 


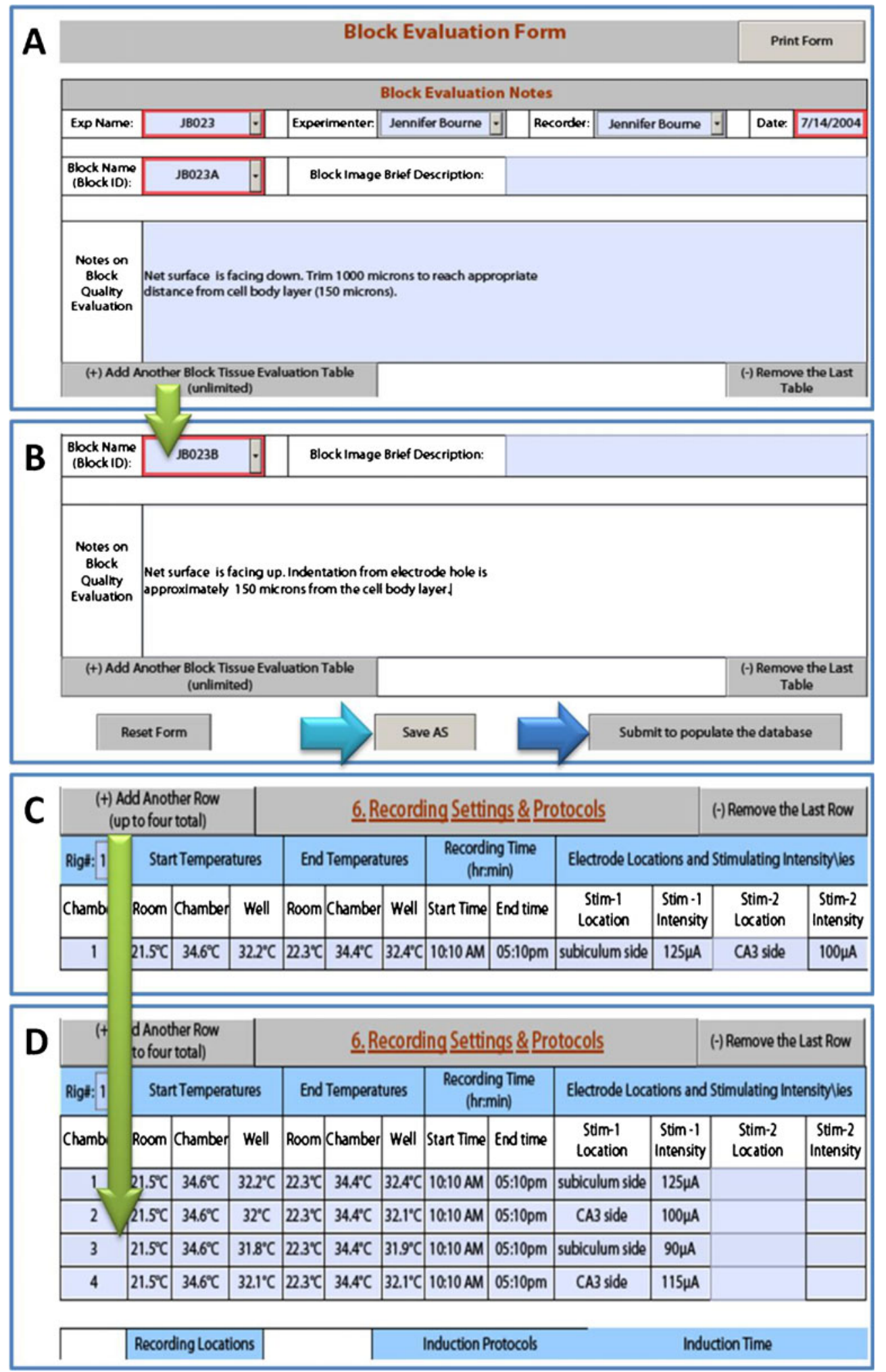

Fig. 5 Example dynamic PDF forms for collecting experimental notes and data. a, b The Block Evaluation Form is for uploading notes regarding information about the orientation of a specific piece of experimental tissue (coded JB023A) in a block of Epoxy resin. An identical second table can be added (green arrow from a to b) in which another block, from the same experiment, can be described (JB023B). Clicking "Save AS" (light blue arrow) is used to save the downloaded PDF form to a local computer, while clicking "Submit to populate the database" button (dark blue arrow) populates the data from this PDF form into the database. c, $\mathbf{d}$ The Dynamic PDF forms also have a feature to "Add another row" to the table (green arrow from $\mathbf{c}$ to $\mathbf{d}$ ), in this example additional information about electrophysiological recordings among different slices from the same animal were entered 


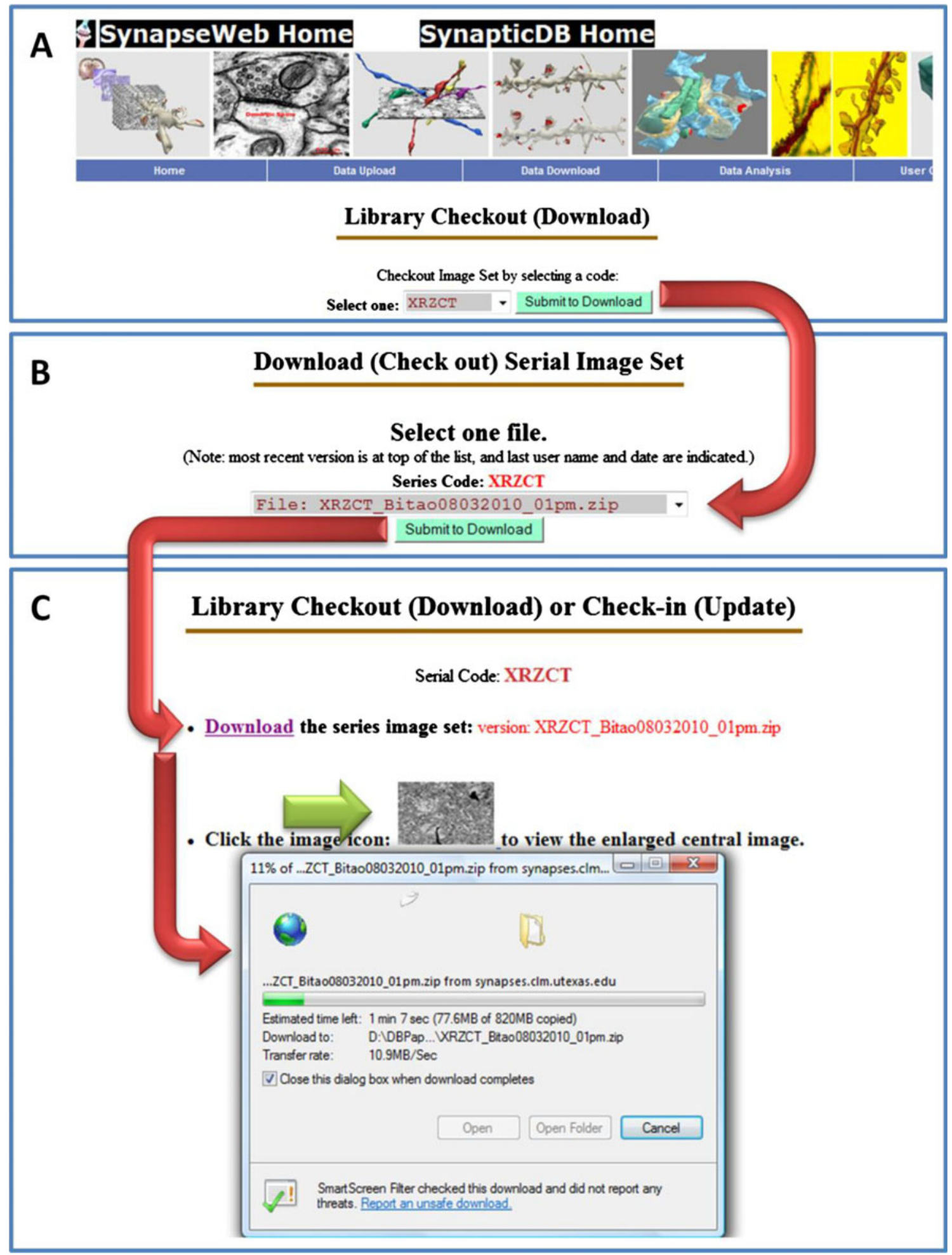

Fig. 6 Using the web interfaces to checkout (download) a set of ssEM image files. a A code name is selected (red arrow from a to b). b If multiple users have contributed to the dataset, then multiple compressed files will be listed by a file name comprising: the code name user name_date appearing in the selection dropdown box. Normally a user would select the most recent file version and click the "Submit to download" button (red arrow from $\mathbf{b}$ to $\mathbf{c}$ ). $\mathbf{c}$ Once the download hyperlink is clicked, the set of serial images is checked out (red arrow) for analysis. Alternatively, the image of the central section of that series can be viewed immediately by clicking its icon (green arrow) 


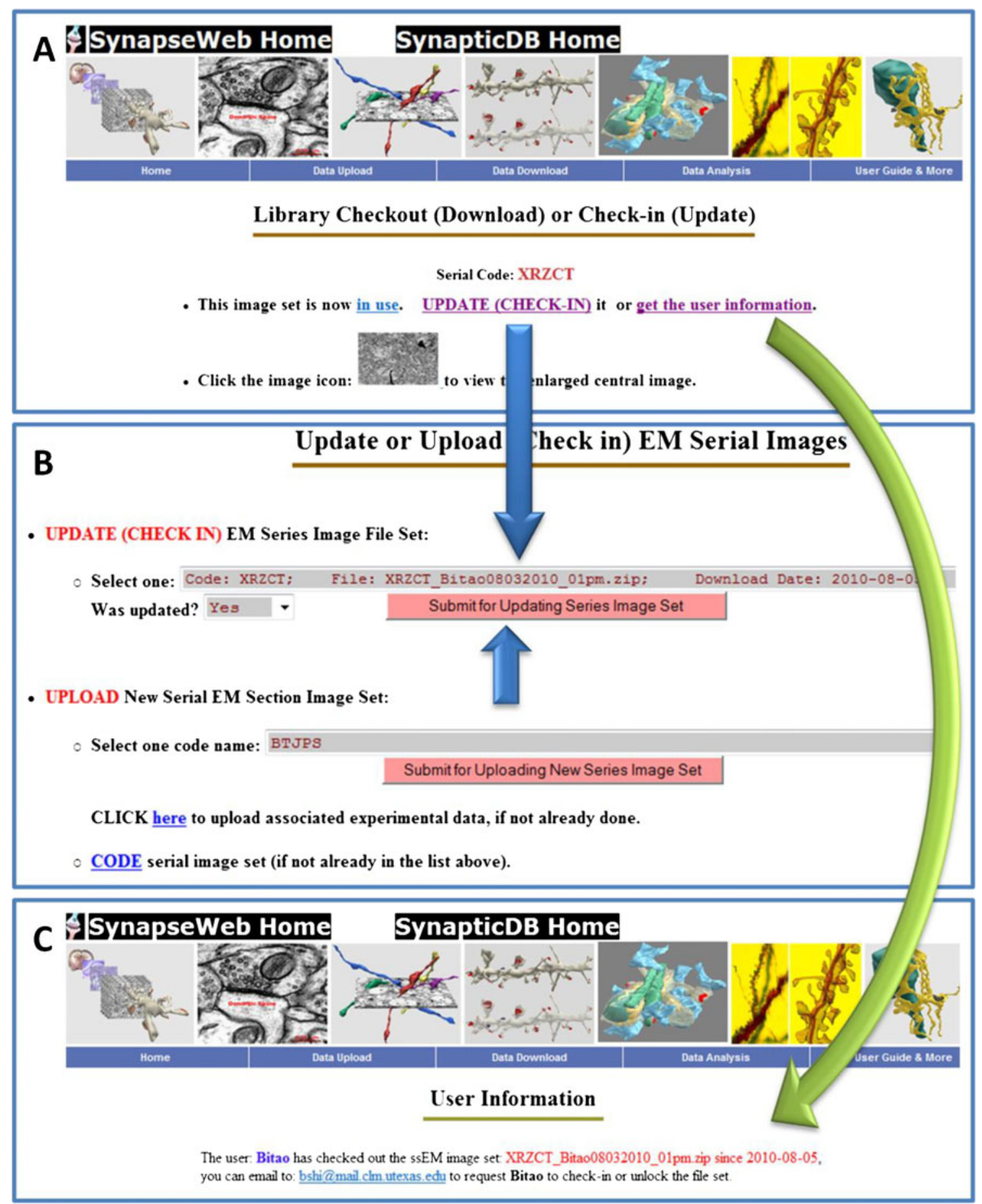

Fig. 7 Web interfaces show how to update or access information about ssEM images that are locked, namely 'in use', after they have been checked out. a First, the desired Serial Code is identified. If the dataset is 'in use' and the user is ready to update it, then the "UPDATE (CHECK IN)" hyperlink is clicked to open the "Check-in' interface (blue arrow from a to b). Alternatively, a user can obtain information about the user who has checked out the dataset by clicking the 'get user information' hyperlink (curved green arrow from a to $\mathbf{c}$ ). b The UPDATE (CHECK-IN) is clicked if the user is ready to check-in a serial image dataset that has been modified, for others to access in Synaptic DB. In "Upload" a user can select a new set of serial images in an existing experiment to upload, or if one got to this window by mistake, there is a Click 'here' link to return to the web interfaces where associated experimental data are entered. Similarly, if the image set does not yet have a unique code, it can be coded by clicking the "CODE" hyperlink. c When the "get" hyperlink is clicked in a, it reveals a web page with the current user's email address and the date when the dataset was checked-out 


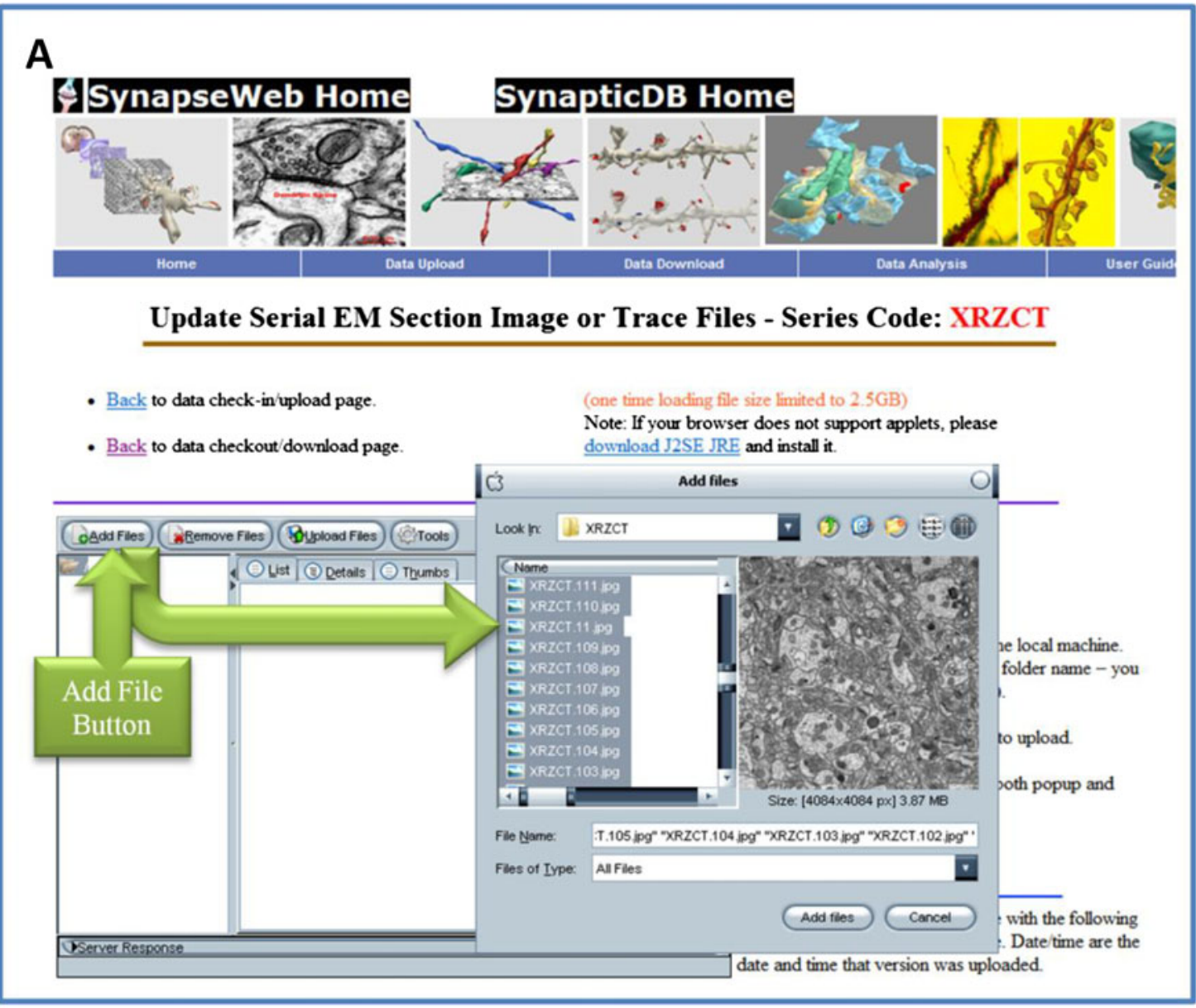

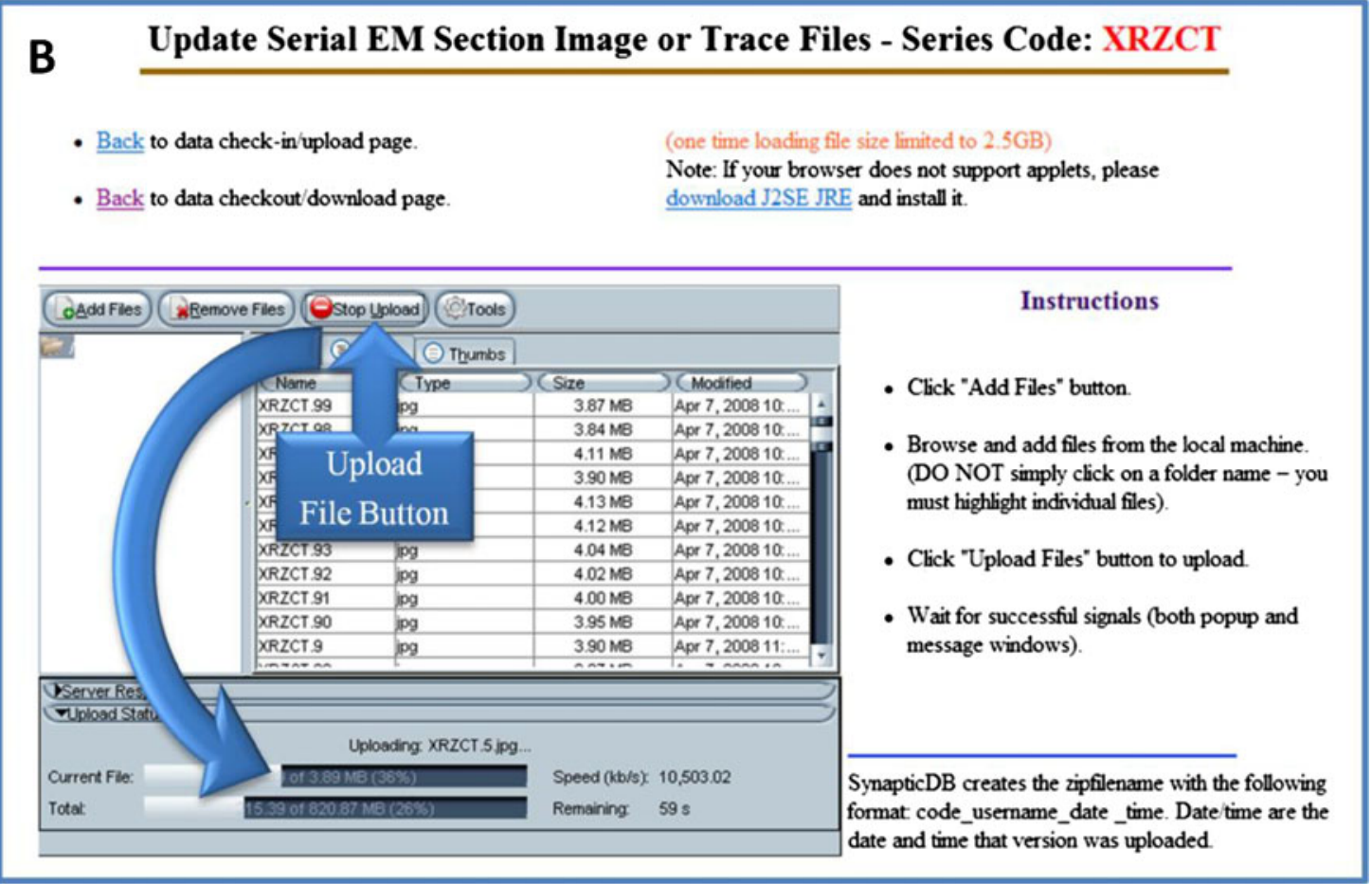

Fig. 8 The web interface with the embedded Java Applet for uploading or removing data files. a The Applet pops up for users to browse files (green arrow callout) and add files (green curved arrow) to a list. b The upload file button (blue arrow callout) is clicked to upload new or check in updated ssEM image data sets (blue curved arrow) 


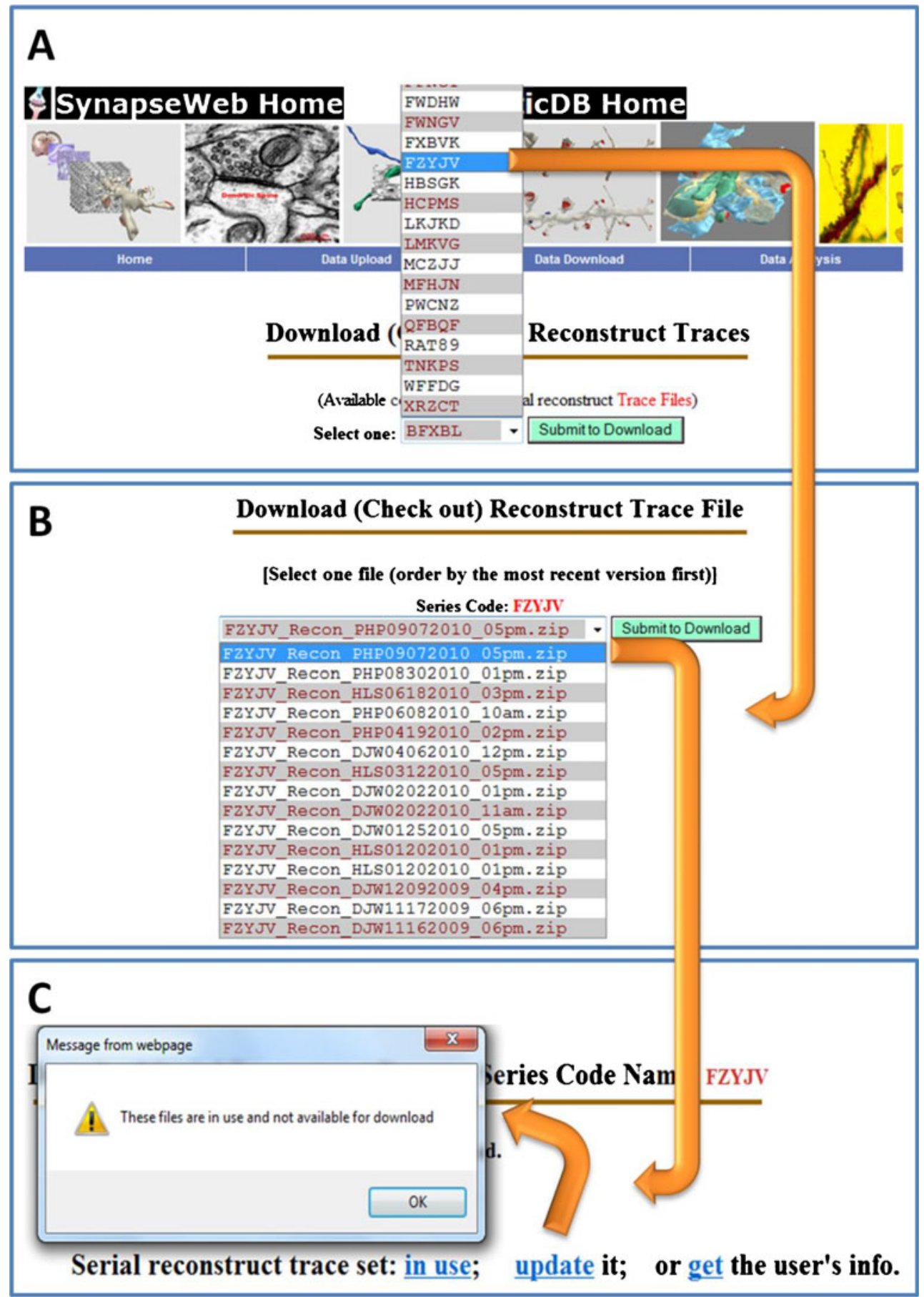

Fig. 9 In this example, the trace files for series FZYJV have been "locked" when in use to prevent duplication of effort across investigators. a Code name and $\mathbf{b}$ most recent set of trace files are selected, but found to be $\mathbf{c}$ in use and not available for download

analysis templates (Fig. 10a). These templates are also used to guide students and other investigators in methods to identify and analyze neuronal, synaptic, and glial structures in ssEM images. Over the years, we have developed numerous templates in the spreadsheet for systematic analyses and these have been populated into SynapticDB (Fig. 10b). Our instructions are located in the comments of cells in the top row and/or left column in spreadsheets. Names, identifications, locations, and quantitative data are filled into the rest of the spreadsheet.

Web-based interfaces have been created in SynapticDB to manage these analysis templates. New templates can be added to the database in spreadsheet format with headers and comments in the top row and/or left column (Fig. 10c). A template can be built from columns and rows already in the database (Fig. 11a), and the output will be a 


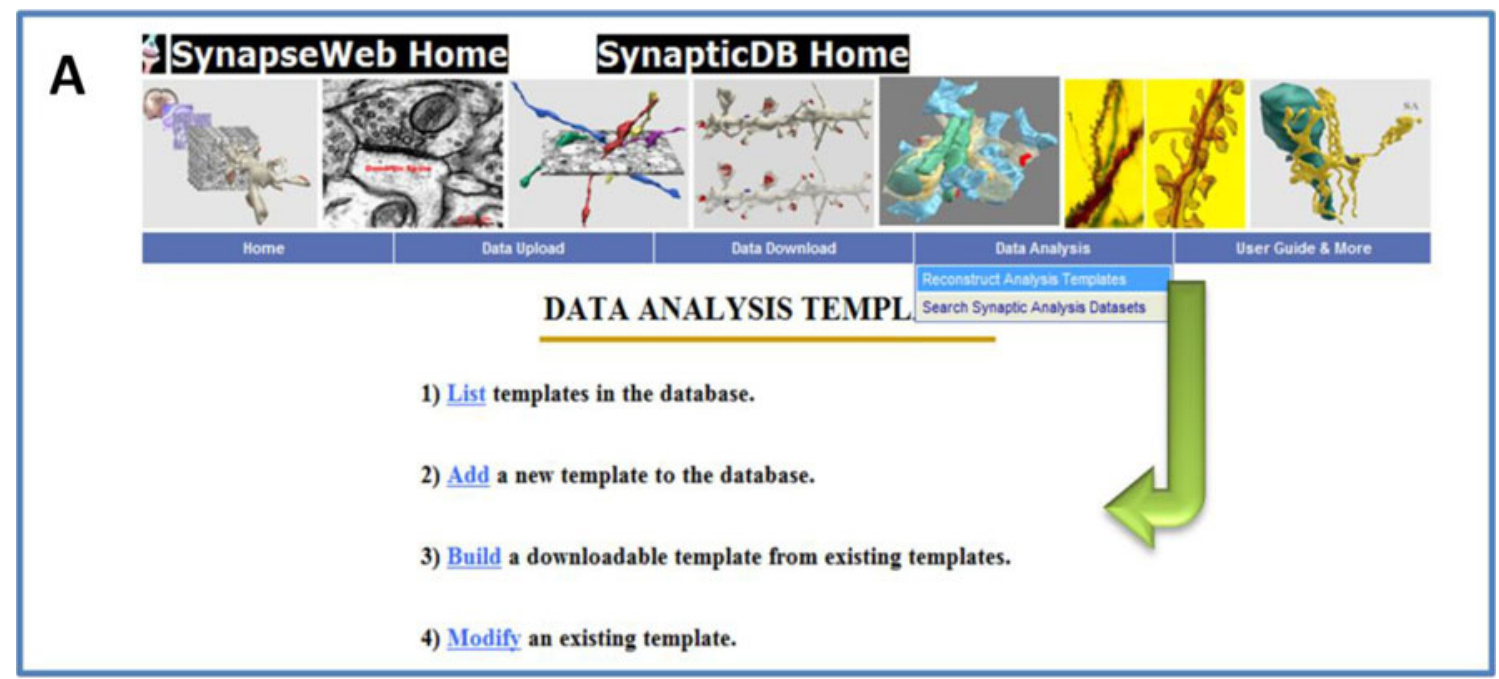

B

\section{DATA ANALYSIS TEMPLATES}

List of Templates in the Database:

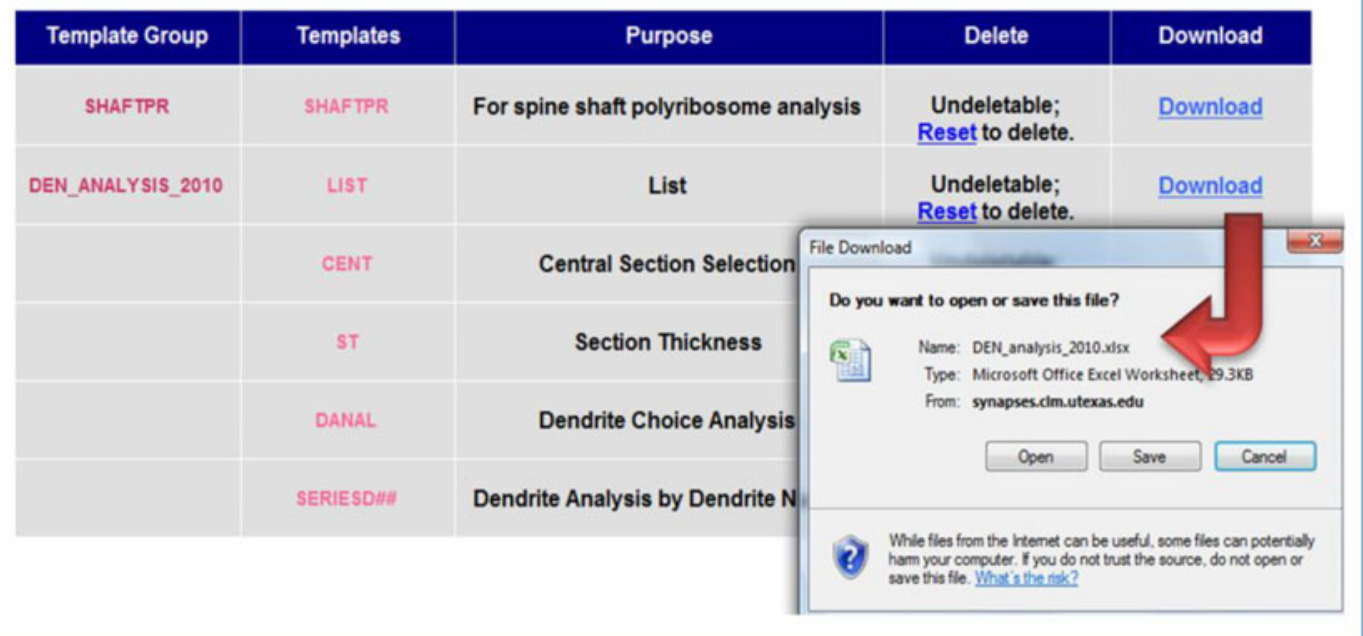

C

\section{DATA ANALYSIS TEMPLATES}

\section{Add a Template to the Database via Excel Spreadsheets:}

(Note: keep all headers and their cmments on the first row and/or first left column of a spreadsheet.)

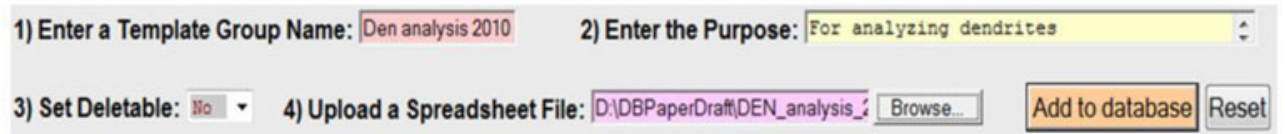

Fig. 10 Web interfaces for managing templates. a Templates can be managed via clicking List, Add, Build and Modify hyperlinks on the web interface. b List shows a subset of the existing and downloadable templates in SynapticDB. A full template can be downloaded and saved in a

downloadable spreadsheet containing the row/column names with instructional header comments positioned in the 'comment' flag in a spreadsheet (Fig. 11b). The comments associated with each header contain instructions spreadsheet file by clicking the download link in the row associated with the desired template group name (red arrow). c Interface used to add new templates to the database by uploading a spreadsheet file where headers and instructional comments are located in the top row and/or left column

on how to measure, identify, or quantify an object across serial sections. The example template shown in Fig. 11 was constructed from three standard column headers for naming polyribosomes found in dendritic shafts (SHPRNAME); 


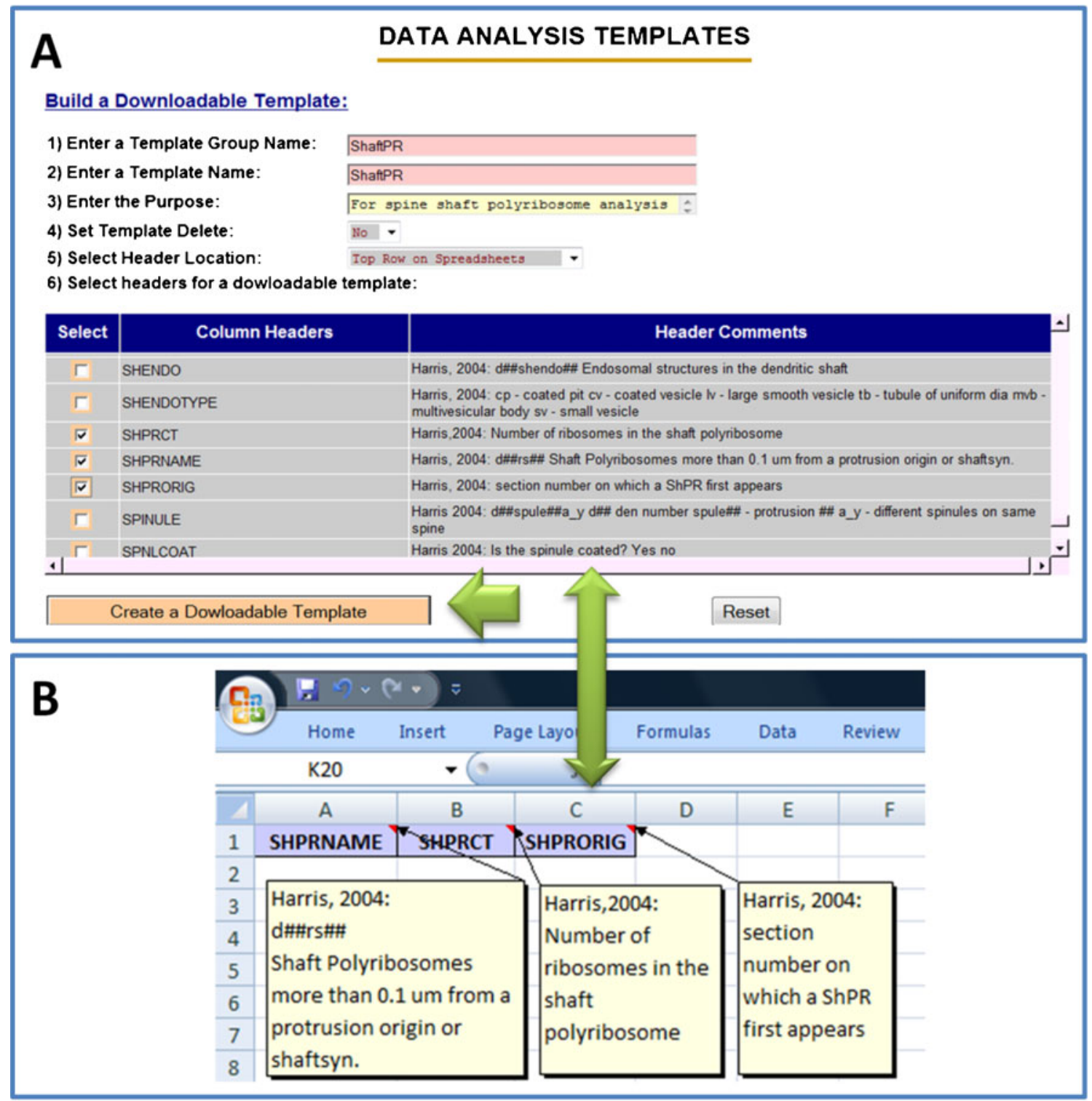

C

\section{DATA ANALYSIS TEMPLATES}

Select One Template to Modify:

\begin{tabular}{|c|c|c|}
\hline Templates & Purpose & Modify \\
\hline 1.SHAFTPR & For spine shaft polyribosome analysis & Mod \\
\hline 2.LIST & List & Modify \\
\hline 3.CENT & Central Section Selection & Modify \\
\hline 4.ST & Section Thickness & Modify \\
\hline 5.DANAL & Dendrite Choice Analysis & Modify \\
\hline 6.SERIESDH\# & Dendrite Analysis by Dendrite Number & Modify \\
\hline
\end{tabular}

Fig. 11 Build or modify a template from components of an existing template. a First, the name of new template group is entered; then the name of the downloadable template that is being built is entered along with its purpose and whether or not it should be deleted after it is built. After they are defined in the top row or left column of a spreadsheet, desired headers with instructional comments are checked and the downloadable template is created by clicking: "Create a Downloadable Template" (green arrow). b The newly built template is downloaded in the spreadsheet file (green arrow from a to b). c To modify an existing template the "Modify" hyperlink is clicked (purple arrow) to evoke the forms in Fig. 12 
counting the number of ribosomes in each polyribosome (SHPRCT) and identifying the section on which they occur (SHPRORG, Fig. 11b). Each template can also be modified by clicking its hyperlink (Fig. 11c). Specific columns in an existing template can be deleted, updated, or new ones can be added (Fig. 12a). To add new columns with each associated header comment, the "Add" hyperlink is invoked (Fig. 12b). Alternatively, an existing column header can be improved with new instructions (Fig. 12c). The system organizes, builds, stores and shares the analysis templates via the implemented parse application of Apache POI API (http://poi.apache.org/). Thus, the template platform implemented in Synaptic DB facilitates preparation of standard analytical templates and provides a mechanism to organize and track their distribution among investigators.

\section{Access to Coded Experimental Data}

SynapticDB also provides experimental data query and retrieval through a systematic data query interface (Fig. 13a). Since each experiment, tissue block and series are uniquely named, corresponding data details in SynapticDB can be retrieved for each specific query. Thus, access interfaces to the database are for series code names (Fig. 13b), tissue block names (Fig. 13c), experiment name (Fig. 13d), or investigator name (Fig. 13e). These queries provide users with multiple access points to obtain any data in the database. Importantly, at the point where an investigator seeks to decode an image series, there are two protections to prevent revealing experimental conditions prematurely, namely a password protection followed by a query to ensure the investigator is ready to decode the experimental conditions for subsequent statistical analyses. If necessary, a new code can later be assigned to the dataset for future analyses and SynapticDB will link it as well as the old code to the appropriate experimental data. Search functions will be added to the system for users to find specific data following keyword entries. These applications are written in Java, deployed in Tomcat engine (http:// tomcat.apache.org/), and hosted on a Windows server.

\section{Discussion}

Ultrastructural study of synapses, dendrites, axons, and glia through ssEM is becoming a mainstay towards understanding brain function. Other Neuroscience database systems have demonstrated that web-enabled tools facilitate analysis, reuse and integration of image data across laboratories (e.g. Toga 2002; Moene et al. 2007; Martone et al. 2004; Marenco et al. 2010, http://ccdb.ucsd.edu/index.shtm; http://neuinfo.org/; http://neurodatabase.org/; http://neuromorpho.org/). SynapticDB is the first integrated system for ssEM studies. It promotes management of the enormous amounts of data generated in ssEM studies, and provides guidance in standardizing analytical strategies. The library mechanism of SynapticDB automatically tracks data transactions and meets the need for systematic online data transfer and sharing, while helping to avoid duplication of effort. At present, SynapticDB is being used and tested extensively by members of the Harris Laboratory and collaborators. In addition, several ssEM image datasets are publically available for download by any registered user without any locking mechanism. All registered users can also access the template facilities and download them for their own research. We also make available a catalogue of 'users' who have downloaded PUBLIC data where they would optionally provide a statement of their intended use and contact information. That way, if another Public user wanted to explore the same question, they could communicate to avoid duplication of effort across laboratories if so desired. SynapticDB currently provides multiple integrated ssEM datasets for public access, and we expect to post more integrated sets of ssEM data as the metadata are collected from past publications, and ongoing studies are completed and published. Already, these have been valuable resources in meta-analyses regarding the composition and circuitry of neurons and synapses (Zhang et al. 2006, 2007; Kinney et al. 2007; Rumsey et al. 2009; Jain et al. 2010; Mishchenko et al. 2010; Kinney et al. 2010).

The ssEM image datasets managed by SynapticDB are also valuable in the development of new automated reconstruction tools. Preparation of excellent tissue for ssEM imaging and collection of the ssEM images is very time consuming and requires a lot of training and skill. An even more important constraint on the advancement of this field is the time required to align, reconstruct and analyze the images manually with reconstruction tools such as RECONSTRUCT ${ }^{\mathrm{TM}}$. Furthermore, while humangenerated reconstructions are usually very accurate with regard to content, the laying down of traces has a subjective component that varies from one person to another, which might impact quantitative values. The field is seeing an unprecedented explosion in effort to improve the speed and accuracy of computer automated alignment, segmentation, reconstruction and analysis tools (Denk and Horstmann 2004; Saalfeld et al. 2010). All of these tools require high quality ssEM images as a starting point (Coggan et al. 2005; Yu et al. 2008; Jain et al. 2010; Mishchenko et al. 2010). Having a public repository of high quality image datasets, with associated manual traces, will greatly facilitate the further development of these computer automated tools. In addition, the maintenance of a library that utilizes different segmentation algorithms to trace the same dendrites, axons, glia, etc., will allow for direct comparisons between recon- 


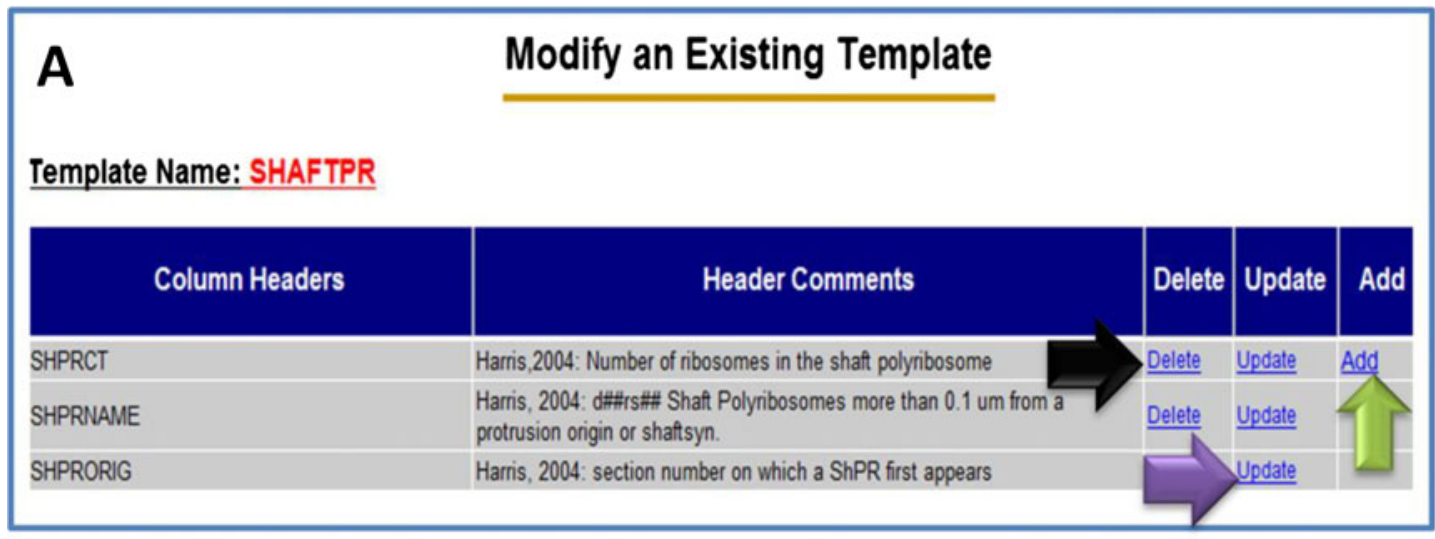

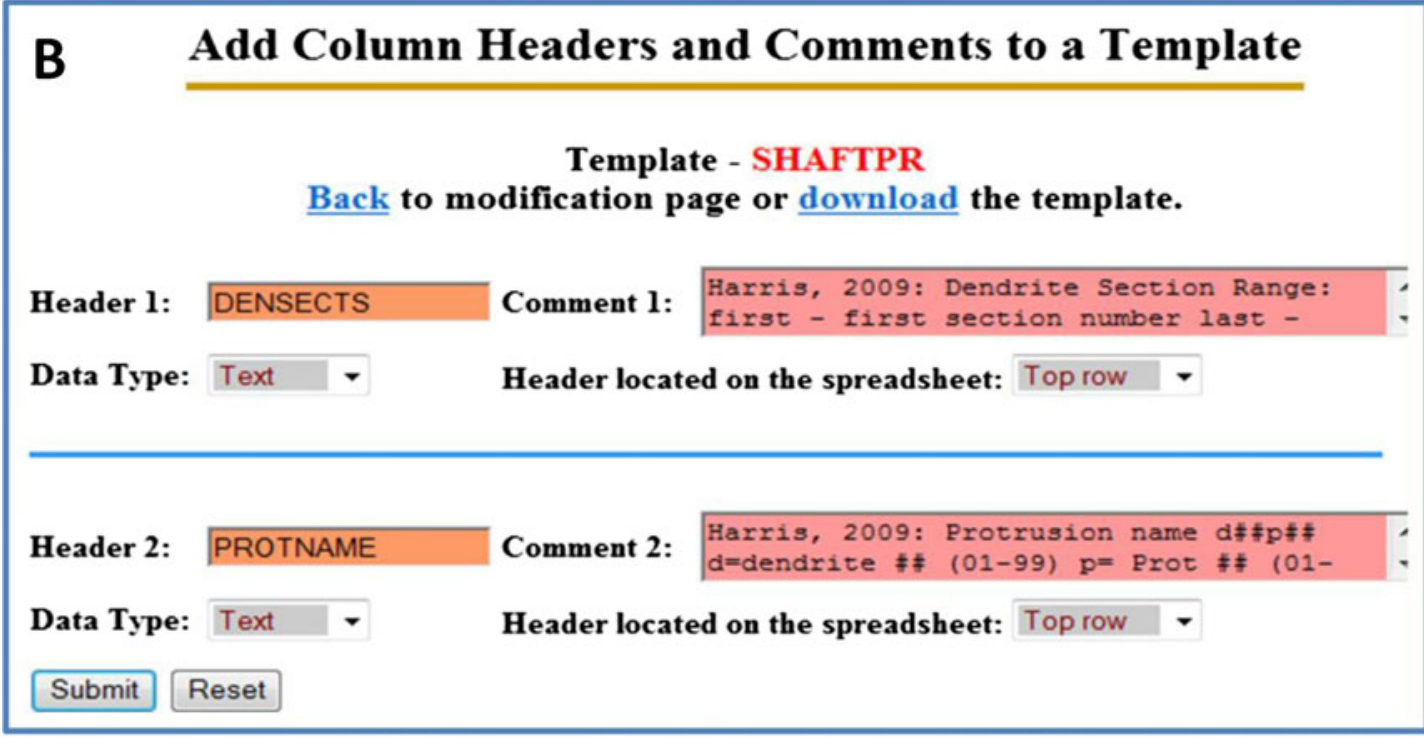

\begin{tabular}{|c|c|c|c|c|}
\hline \multicolumn{5}{|c|}{ Update Template Headers and Comments } \\
\hline \multirow[b]{2}{*}{ Current Header: } & ack to modific & \multirow[t]{3}{*}{$\begin{array}{l}\text { mplate: SHAFTPR } \\
\text { on page or downlos }\end{array}$} & the template. & \\
\hline & SHPRORIG & & \multirow[t]{2}{*}{$\begin{array}{l}\text { Harris, } 2004 \text { : } \\
\text { section number on which a ShPR first } \\
\text { appears }\end{array}$} & \multirow[t]{2}{*}{$\begin{array}{l}\hat{E}] \\
\dot{[}\end{array}$} \\
\hline Current Data Type: & Text & & & \\
\hline New Header: & ShPROri & \multirow[t]{3}{*}{ New Comment: } & \multirow[t]{3}{*}{$\begin{array}{l}\text { Harris, 2010: } \\
\text { section number on which a ShPR first } \\
\text { appears }\end{array}$} & \multirow[t]{3}{*}{$\begin{array}{l}\hat{E} \\
\text { - }\end{array}$} \\
\hline New Data Type: & Text & & & \\
\hline Submit & & & & \\
\hline
\end{tabular}

Fig. 12 Interface to modify column headers and annotations in an existing template. a any item can be added to the template by clicking its "Add" (green arrow). Any item in the template can be modified by clicking its "delete" (black arrow) or "update" (purple arrow) hyperlinks. b Interface for adding more items to the existing template.
The "Data Type" dropdown box provides text or numeric options. The "Headers located on the spreadsheet" dropdown box has options Top row or Left column to define item location in the modified template. c Interface for updating current items in an existing template 

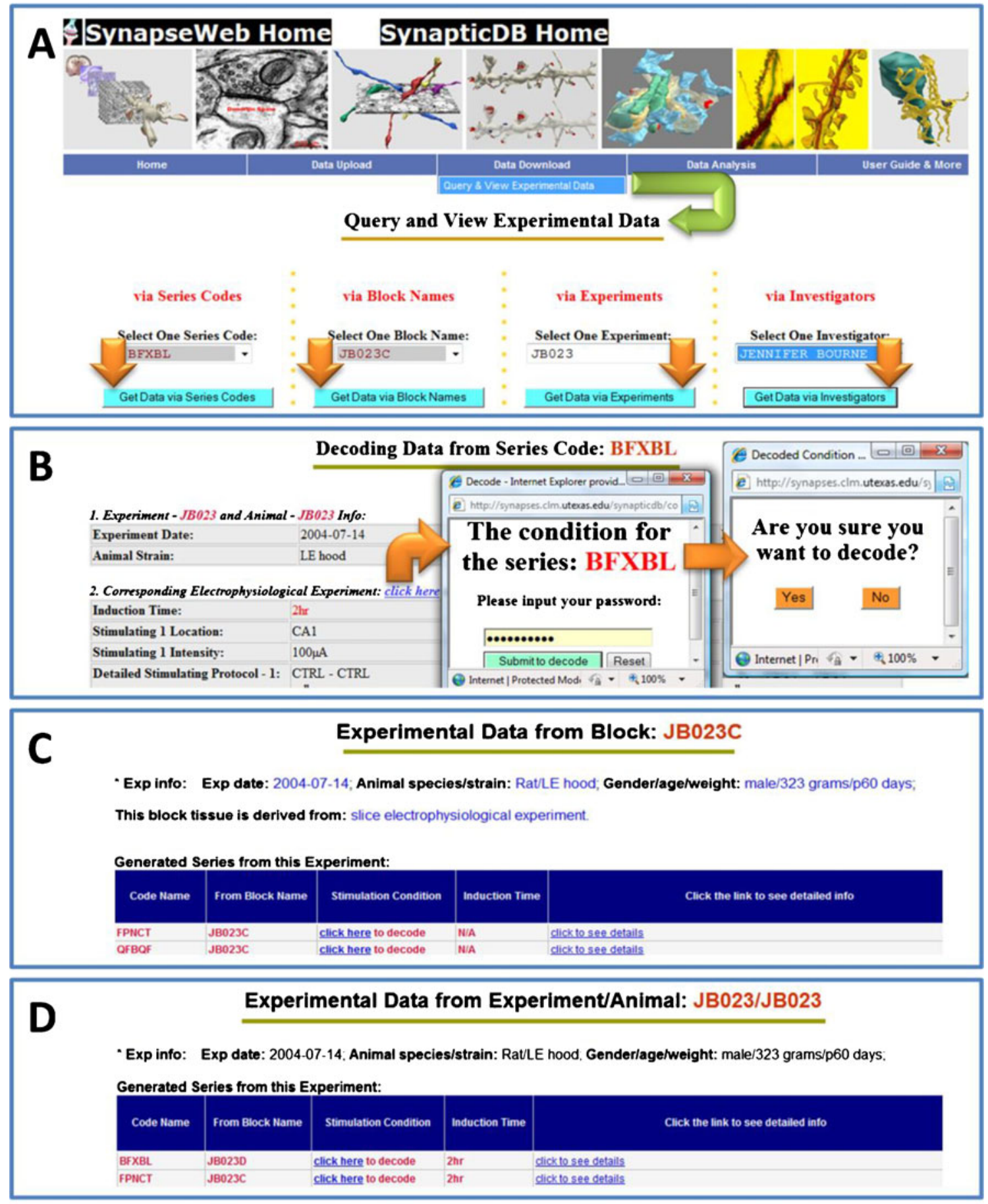

\begin{tabular}{|c|c|c|}
\hline & \multicolumn{2}{|c|}{ Investigator: JENNIFER BOURNE has following experiments } \\
\hline & \multicolumn{2}{|l|}{ Experiment List: } \\
\hline & Experiment Mames & Click the link to see detailed into \\
\hline & J8023 & click to see details from this experiment \\
\hline & J8024 & click to see details from this experiment \\
\hline
\end{tabular}

Fig. 13 Interfaces to query and to decode data. a The data retrieval can be initiated by using code, experiment, block or investigator names (orange arrows). b The queried experiment notes and data are displayed via a code name. The pop up window provides password protected queries to ensure that the investigator is ready to reveal the experimental condition (orange arrows). c, d, e Display of the experimental data via block, experiment or investigator names, respectively 
structions generated by manual tracing and various automated tools.

SynapseWeb (http://synapses.clm.utexas.edu), the home of SynapticDB, provides detailed ultrastructural tutorials and an atlas of ultrastructural neurocytology, which serve to identify various components of neurons and glia. SynapticDB further serves the Neuroscience community by providing standard analytical templates for consistency in entering identities and quantifying ultrastructure through ssEM. Furthermore, uploading data in these formats provides instant and remote population of the database, which should facilitate communication and sharing of information between experienced and novice investigators. SynapticDB provides the infrastructure for the future development of search engines and metaanalyses of the data as it is acquired.

\section{Conclusions}

1. SynapticDB provides a practical and effective ssEM data management system that both stores and allows managed viewing and sharing of the data. 2 . The embedded, prepopulated, dynamic and stand alone PDF forms provide convenient management, collection and uploading of supporting experimental notes and data. 3. Importantly, there is a coding feature that allows investigators to remain blind as to specific experimental conditions while analyzing images, thereby reducing potential experimenter biases. 4 . The library checkout and check-in data management mechanism, with its locking capability, facilitates data sharing within a laboratory while avoiding duplication in effort. 5. The remotely accessed and standardized instructional analysis templates educate novice investigators and promote consistency in analytical strategies across investigators. 6. SynapticDB provides the infrastructure to collect, search, and perform meta-analysis of neuroscience data at the nanometer scale of synapses. 7. There are datasets publically available on SynapticDB that have been used towards successful image analysis and publication of new findings.

\section{Information Sharing Statement}

Several ssEM image datasets can be downloaded by any registered user via "http://synapses.clm.utexas.edu/synapticdb/ index pub.jsp". In addition, all registered users can access the library and template facilities for use in their own research.

Acknowledgements We thank all members in the Harris laboratory for editing experimental collection forms and testing the system. The work was supported by NIH grants EB002170 and NS021184.
Open Access This article is distributed under the terms of the Creative Commons Attribution Noncommercial License which permits any noncommercial use, distribution, and reproduction in any medium, provided the original author(s) and source are credited.

\section{References}

Amari, S. I., Beltrame, F., Bjaalie, J. G., et al. (2002). Neuroinformatics: the integration of shared databases and tools towards integrative neuroscience. Journal of Integrative Neuroscience, 1, $117-128$.

Bjaalie, J. G., \& Leergaard, T. B. (2005). Three-dimensional visualization and analysis of wiring patterns in the brain: experiments, tools, models, and databases. In: Koslow, S. H. \& Subramaniam, S. (Eds.), Databasing the brain: From data to knowledge (pp. 349-368). New York: Wiley.

Coggan, J. S., Bartol, T. M., Esquenazi, E., Stiles, J. R., Lamont, S., Martone, M. E., et al. (2005). Evidence for ectopic neurotransmission at a neuronal synapse. Science, 309, 446-451.

Crabbe, J. C., \& Morris, R. G. M. (2004). Festina lente: late-night thoughts on high-throughput screening of mouse behavior. Nature Neuroscience, 7(11), 1175-1179.

Davison, A. P., Morese, T. M., Migliore, M., Shepherd, G. M., \& Hines, M. L. (2004). Semi-automated population of an online database of neuronal models (ModelDB) with citation information, using PubMed for validation. Nueroinformatics, 2, 327-332.

Denk, W., \& Horstmann, H. (2004). Serial block-face scanning electron microscopy to reconstruct three-dimensional tissue nanostructure. PLoS Biology, 2, e329.

Fiala, J. C. (2005). Reconstruct: a free editor for serial section microscopy, J. Microscopy, 218, 52-61.

Fiala, J. C., \& Harris, K. M. (2001). Extending unbiased stereology of brain ultrastructure to three-dimensional volumes. Journal of the American Medical Informatics Association, 8(1), 1-16.

Gardner, D., Abato, M., Knuth, K. H., \& Robert, A. (2005). Neuroinformatics for neurophysiology: the role, design, and use of databases. In: Koslow, S. H. \& Subramaniam, S. (Eds.), Databasing the brain: From data to knowledge (pp. 47-67). New York: Wiley.

Gardner, D., Akil, H., Ascoli, G. A., Bowden, D. M., Bug, W., Donohue, D. E., et al. (2008). The Neuroscience Information Framework: a data and knowledge environment for neuroscience. Neuroinformatics, 6(3), 149-160.

Jain, V., Bollmann, B., Richardson, M., Berger, D., Helmstaedter, M., Briggman, K., et al. (2010). Boundary learning by optimization with topological constraints. IEEE Conference on Computer Vision and Pattern Recognition, 2010, 2488-2495.

Kinney, J. P., Spacek, J., Bartol, T. M., Bajaj, C., Harris, K. M., \& Sejnowski, T. J. (2007). Effect of extracellular space width on geometric tortuosity in 3D reconstruction of neuropil. Society for Neuroscience Abstracts, 360.19.

Kinney, J. P., Bartol, T. M., Regner, B., Bajaj, C. L., Harris, K. M., \& Sejnowski, T. J. (2010). The 3D structure of hippocampal extracellular space has sheets and tunnels. Society for Neuroscience Abs. 853.1.

Laird, A. R., Lancaster, J. L., \& Fox, P. T. (2005). The social evolution of a human brain mapping database. Neuroinformatics, 3, 65-77.

Li, J., Ning, Y., Hedley, W., et al. (2002). The molecule pages database. Nature, 420, 716-717.

Liu, N., Xu, F., Marenco, L., Hyder, F., Miller, P., \& Shepherd, G. M. (2004). Informatics approaches to functional MRI odor mapping of the rodent olfactory bulb OdorMapBuilder and OdorMapDB. Neuroinformatics, 2, 3-18. 
Marenco, L., Wang, R., Shepherd, G. M., \& Miller, P. L. (2010). The NIF DISCO framework: facilitating automated integration of neuroscience content on the web. Neuroinformatics, 8(2), 101-112.

Martone, M. E., Zhang, S., Gupta, A., Qian, X., He, H., Price, D. L., et al. (2003). The cell-centered database: a database for multiscale structural and protein localization data from light and electron microscopy. Neuroninformatics, 1, 379-395.

Martone, M. E., Gupta, A., \& Ellisman, M. H. (2004). E-Neuroscience: challenges and triumphs in integrating distributed data from molecules to brains. Nature Neuroscience, 7(50), 467-472.

Mishchenko, Y., Hu, T., Spacek, J., Mendenhall, J., Harris, K. M., \& Chklovskii, D. B. (2010). Ultrastructural analysis of hippocampal neuropil from the connectomics perspective. Neuron, 67(6), $1009-1020$

Moene, I. A., Subramaniam, S., Darin, D., Leergaard, T. B., \& Bjaalie, J. G. (2007). Toward a workbench for rodent brain image data systems architecture and design. Neuroinformatics, 5, 35-57.

Nesvold, H. (2007). Implementing e-forms with Adobe LiveCycle Forms. TheServerSide.com, Sept. 1 (http://www.theserverside.com/news/ 1364115/Implementing-e-forms-with-Adobe-LiveCycle-Forms).

Nielsen, F. A., Hansen, L. K., \& Balslev, D. (2004). Mining for associations between text and brain activation in a functional neuroimaging database. Neuroinformatics, 2, 369-379.

Pittendrigh, S., \& Jacobs, G. (2003). NeuroSys: a semistructured laboratory database. Neuroinformatics, 1, 167-176.

Rosen, G. D., La Porte, N. T., Diechtiareff, B., et al. (2003). Informatics center for mouse genomics: the dissection of complex traits of the nervous system. Neuroinformatics, 1, 327-342.
Rumsey, C., Gillette, A., Bettadapura, R., Mollere, A., Kinney, J. P., Bartol, T. M., et al. (2009). Reconstructing the neuropil from serial section transmission electron microscopy. Society for Neuroscience Abstracts, 687.2.

Saalfeld, S., Cardona, A., Hartenstein, V., \& Tomancák, P. (2010). As-rigid-as-possible mosaicking and serial section registration of large ssTEM datasets. Bioinformatics, 26, i57-i63.

Tecott, L. H., \& Nestler, E. J. (2004). Neurobehavioral assessment in the information age. Nature Neuroscience, 7(5), 462-466 (Behavior data)

Toga, A. W. (2002). Neuroimage databases: the good, the bad and the ugly. Nature Reviews. Neuroscience, 3, 302-309.

Van Horn, J. D., Grafton, S. T., Rockmore, D., \& Gazzaniga, M. S. (2004). Sharing neuroimaging studies of human cognition. Nature Neuroscience, 7(5), 473-481.

Yu, Z., Holst, M. J., Hayashi, T., Bajaj, C. L., Ellisman, M. H., McCammon, J. A., et al. (2008). Three-dimensional geometric modeling of membrane-bound organelles in ventricular myocytes: bridging the gap between microscopic imaging and mathematical simulation. Journal of Structural Biology, 164, 304-313.

Zhang, W., Bartol, T. M., Harris, K. M., \& Sejnowski, T. J. (2006). Monte Carlo model of presynaptic calcium dynamics of a hippocampal neuron. Society for Neuroscience Abstracts, 338.12.

Zhang, W., Bartol, T. M., Harris, K. M., \& Sejnowski, T. J. (2007). Monte Carlo study of calcium dynamics in a Schaeffer collateral multiple-synapse bouton. Society for Neuroscience Abstracts, 359.10. 